\title{
INTRODUCTION \\ New techniques and technologies in the management of ischemic stroke
}

\author{
William J. Mack, MD, ${ }^{1}$ Louis J. Kim, MD, ${ }^{2}$ Demetrius K. Lopes, MD, ${ }^{3}$ and J Mocco, MD, MS ${ }^{4}$ \\ 1Department of Neurosurgery, University of Southern California, Los Angeles, California; ${ }^{2}$ Department of Neurosurgery, \\ University of Washington, Seattle, Washington; ${ }^{2}$ Department of Neurosurgery, Rush University Medical Center, Chicago, Illinois; \\ and ${ }^{4}$ Department of Neurosurgery, Mt. Sinai School of Medicine, New York, New York
}

$\Lambda$ CUTE stroke treatment has evolved rapidly in the past decade. Once a medical disease, emergent large-vessel occlusions are now routinely and effectively treated by neuroendovascular surgeons. Five landmark trials reported in the New England Journal of Medicine in 2015 drastically changed the landscape of acute stroke treatment. Mechanical thrombectomy is now the standard of care. This new era has ushered in rapid advances and innovations that cover the spectrum of acute stroke management. Improvements in imaging protocols, systems of care, procedural techniques, and new device technologies continue to shape a rapidly expanding and evolving field.

This issue of Neurosurgical Focus aims to deliver an overview of the latest developments in acute stroke care. Articles provide the reader with information and insight into advances along the entire clinical-translational spectrum-from basic science and novel animal models to clinical implementation and stroke systems of care.

The first set of articles provides an in-depth review of neuroprotective strategies and their potential utility as adjuncts to mechanical thrombectomy. Successful coupling of these modalities could ultimately extend the window for effective endovascular stroke treatment. Next, a novel animal model of recalcitrant, large clot burden, bifurcation occlusion for mechanical thrombectomy is presented. The following group of articles explores emerging technologies and systems of care to improve access to endovascular stroke treatment. While neuroendovascular surgeons have become adept at recanalizing occluded vessels, the ability to triage appropriate patients to capable treatment centers remains a challenge. The final group of articles addresses new developments in imaging capabilities and operative techniques that are advancing mechanical thrombectomy procedures and perioperative care.

We thank the authors who contributed to this issue. We also thank the staff of Neurosurgical Focus for the privilege of participating in the design and execution of this issue and for their skill and assistance in the editing and production process.

https://thejns.org/doi/abs/10.3171/2017.1.FOCUS1730

\section{Disclosures}

Dr. Lopes reports a consultant relationship with Penumbra and Siemens and receipt of clinical or research support from Medtronic, Stryker, and MicroVention. Dr. Mocco reports a consultant relationship with Cerebrotech, Rebound Medical, The Stroke Project, Endostream, Pulsar, Synchron, and Vastrax and ownership interest in NeuroTechnology Investors, Cerebrotech, Apama Medical, Cosmo, The Stroke Project, Comet, Endostream, and Synchron. 